\title{
ORION PROPLYDS AND THE EAGLE'S EGGS
}

Observing the effects of high-mass star formation on low-mass neighbours

\author{
MARK MCCAUGHREAN \\ Max-Planck-Institut für Radioastronomie, \\ Auf dem Hügel 69, 53121 Bonn, Germany
}

\begin{abstract}
We describe the effects of environment on low-mass star formation, from the perspective that most stars in the galaxy form in dense clusters and aggregates in GMCs, often in the presence of massive OB stars. First, we discuss the observational evidence for circumstellar disks around stars of the Trapezium Cluster, and how they are affected by their environment. Second, we examine the more distant M 16, where photo-evaporation by OB stars may disrupt the star formation process, and may inhibit the development of circumstellar disks.
\end{abstract}

\section{Introduction}

Jets and outflows from young stars play an important role in regulating angular momentum removal and dissipation of the outer envelope, and thus perhaps defining parameters such as the final stellar mass. In addition, the outflow may stir up the surrounding molecular cloud on scales up to parsecs, thus affecting further collapse and star formation in the cloud. However, our impression of the global importance of such effects can be biased by the fact that the most beautiful (and thus most studied) examples of collimated jets and outflows are generally associated with more or less isolated stars, in relatively quiescent regions such as the Taurus-Auriga dark clouds, or in the farther reaches of the Orion giant molecular clouds. In those regions, star formation is occuring at a low density, with only $\sim 0.3-3 \mathrm{stars} / \mathrm{pc}^{2}$ (cf. Gomez et al. 1993 for Taurus-Auriga). In contrast, most stars in the galaxy form in GMCs in dense aggregates or clusters of 10-1000 members, with only a minority spread throughout the cloud in a "pedestal" population (Lada et al. 1993; Zinnecker et al. 1993). Within these clusters, the stellar density is much greater, $\sim 10^{3}-10^{4} \mathrm{stars} / \mathrm{pc}^{3}$, implying interactions between stars in the protostellar phase, with adjacent cloud cores compet- 
ing for material, and later, when star-disk systems may collide. Also, many clusters contain $\mathrm{OB}$ stars which, with their ionizing radiation and strong winds, add another level of disruption to their low-mass neighbours, typically only $0.01-1 \mathrm{pc}$ away. The cumulative effects of cluster life are, as yet, relatively poorly understood, but departure from the relative quiescence of the isolated star formation paradigm may, in fact, play a crucial role in determining important global parameters such as the stellar inital mass function and the fraction of stars which form planetary systems.

\section{Circumstellar disks in the Orion Nebula}

The most prominent nearby site of on-going high- and low-mass star formation is in the Orion GMCs at $\sim 500 \mathrm{pc}$. There are concentrated sites of star formation in the clouds, as well as a pedestal, distributed population of young stars. Orion B (L 1630) contains dense clusters of up to 200 stars associated with NGC 2024, NGC 2023, NGC 2068, and NGC 2071, and a near-IR survey of the cloud showed that at least $60 \%$ (and up to $90 \%$ after field star corrections) of the total stellar population is in those clusters (Lada et al. 1991). Similarly, a survey of much of the southern half of the Orion A (L 1641) cloud concluded that roughly half of the stars are in dense aggregates or small clusters of up to 20 members (Strom et al. 1993; Allen 1996). The Orion A cloud also includes the Orion Nebula H II region, inside which is the Trapezium Cluster of up to 1000 young stars (Herbig \& Terndrup 1986; Zinnecker et al. 1993; Prosser et al. 1994; McCaughrean \& Stauffer 1994; Hillenbrand 1997), and thus, averaged over the whole cloud, cluster-mode star formation again dominates. The Trapezium Cluster is a particularly important laboratory for studying the effects of dense clusters on circumstellar disks.

\subsection{DETECTION VIA IR EXCESS}

Far-IR and millimetre excesses are a clear signature of a circumstellar disk, but the limited spatial resolution of space-based IR satellites and single dish millimetre telescopes makes it impossible to measure conventional SEDs for stars in the Trapezium Cluster, typically only a few arcsec apart. However, the hot inner edges of disks radiate at $2-10 \mu \mathrm{m}$, where ground-based IR imaging can separate the stars. Infrared imaging of the cluster from 1$4 \mu \mathrm{m}$ shows that $\sim 60-80 \%$ (depending on the reddening vector for the region) of the Trapezium Cluster stars show excess $3.4 \mu \mathrm{m}$ emission typical of the inner edge of a disk (McCaughrean et al. 1996; Kenyon \& Hartmann 1995). Mid-IR imaging of a few select sources confirms that these excesses extend to $12 \mu \mathrm{m}$, and that the optical-IR SEDs can be fit with a standard disk model (Hayward \& McCaughrean 1997). The implication then is that 
disks are common in the cluster, but we must keep in mind that 2-10 $\mu \mathrm{m}$ emission only probes material within 1-2 AU of the central star: a canonical circumstellar disk capable of producing a planetary system similar to our own (30 AU radius) would need to be much larger.

\subsection{DETECTION AS IONIZED PROPLYDS}

The core of the Trapezium Cluster is dominated by the eponymous Trapezium OB stars, the most massive of which, $\theta^{1}$ Ori C, is an $\mathrm{O} 6$ star responsible for ionizing the Orion Nebula, as well as a number of compact $(\sim 1$ arcsec, $500 \mathrm{AU}$ diameter) nebulae discovered through ground-based optical and radio imaging (Laques \& Vidal 1979; Garay et al. 1987; Churchwell et al. 1987; Felli et al. 1993), and since studied in great detail with the HST (O'Dell et al. 1993; Stauffer et al. 1994; O'Dell \& Wen 1994; Bally et al. 1995; O'Dell \& Wong 1996).

The mass of these nebulae can be crudely estimated by converting the radio continuum flux into a mass-loss rate $\left(\sim 10^{-7} M_{\odot} /\right.$ yr $)$, and multiplying by the age of the cluster $\left(\sim 10^{6} \mathrm{yrs}\right)$ to give a reservoir of $\sim 0.1 M_{\odot}$ (Churchwell et al. 1987; Stauffer etal. 1994). Since optical-IR stars are visible in almost every one of these nebulae (McCaughrean \& Stauffer 1994; O'Dell \& Wong 1996), the underlying reservoir of material must be in the form of a disk (Churchwell et al. 1987). Accordingly, these sources are thought to be externally ionized proto-planetary disks or "proplyds" (O'Dell et al. 1993). Initial models of such sources are able to reproduce some of the key observational features (Henney et al. 1996; Johnstone et al. 1996).

The disks are not generally directly observed in the proplyds; their presence is inferred by contrasting the mass and size of the reservoir with the visibility of the central star. However, the reservoir mass may be much lower than presumed. First, the mass-loss rate is calculated by assuming that the ionized gas is flowing freely away: if it is actually trapped, the mass-loss rate will be lower. Second, the timescale over which a proplyd is exposed to the ionizing radiation from $\theta^{1}$ Ori $C$ may be much shorter than $10^{6}$ yrs: the OB stars may have formed later than the surrounding low-mass stars and may be only $\sim 10^{5} \mathrm{yrs}$ old, while the $1-2 \mathrm{~km} / \mathrm{s}$ velocity dispersion of the cluster will cycle the low-mass stars in and out of the core on timescales of $10^{5} \mathrm{yrs}$. If the mass-loss rate and duration of exposure to ionizing radiation were both an order of magnitude lower, a reservoir mass as small as $0.001 M_{\odot}$ would be inferred, more consistent with direct observations at millimetre wavelengths which indicate masses $\sim 0.005 M_{\odot}$ or lower (Mundy et al. 1996; Lada, personal communication). Such low reservoir masses would not necessarily invalidate the conclusion that they are in the form of disks, since the optical visibility of the central stars places a stringent constraint on the 

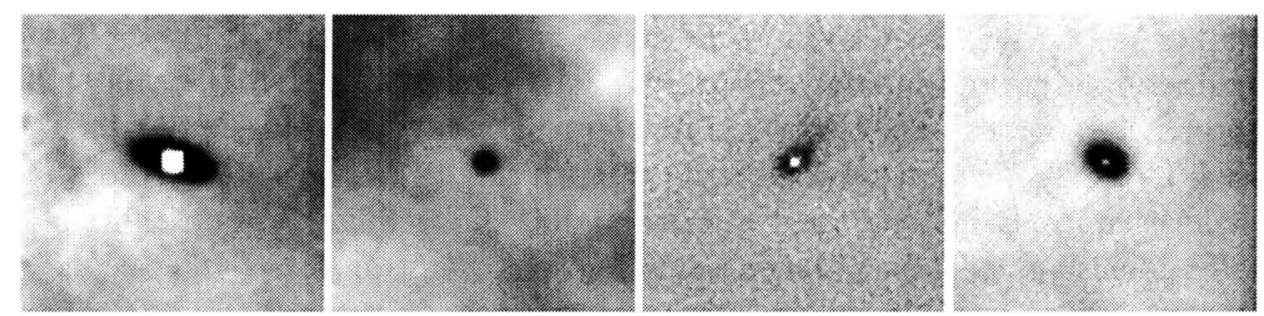

Figure 1. HST images of four silhouette disks in the Orion Nebula. From left to right: Orion 218-354, 167-231, 121-1925, and 183-405. All are seen against the bright $\mathrm{H} \alpha$ background of the nebula. Each panel is 4.1 arcsec or $1800 \mathrm{AU}$ square, $c f$. the $60 \mathrm{AU}$ diameter of the Solar System.
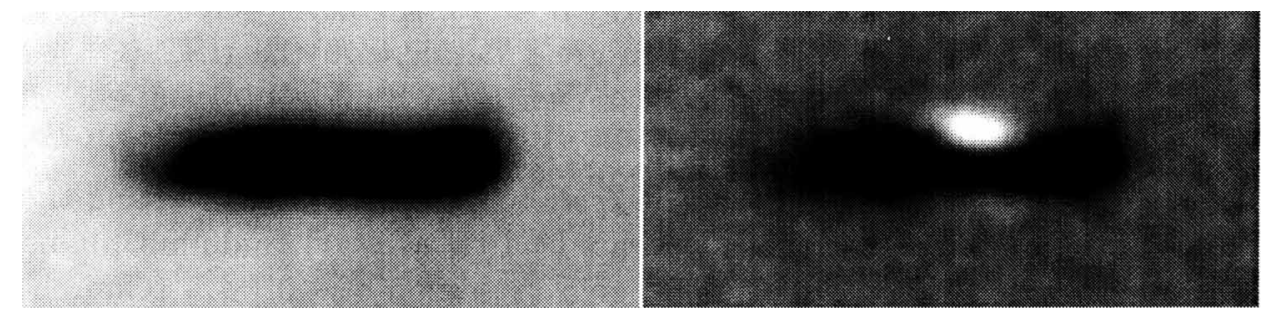

Figure 2. HST images of the Orion 114-426 near edge-on disk. The right panel shows the disk clearly silhouetted against the nebular $\mathrm{H} \alpha$ background. The left image shows an F547M continuum image, in which faint reflection nebulae are seen above and below the plane of the disk. Each panel is $1800 \times 900 \mathrm{AU}$ in size.

geometry. However, these disks would be an order of magnitude less massive than the minimum mass solar nebula, and thus unlikely to form planetary systems similar to our own. Therefore, although the ionized proplyds probably contain disks, it is not clear if they are true proto-planetary disks: more accurate mass estimates are needed via millimetre interferometry.

\subsection{DETECTION AS SILHOUETTES}

In special cases, a disk may be imaged directly as a silhouette against the bright background emission of the Orion Nebula, and a small number of such sources have been found (Figures 1 and 2; McCaughrean \& O'Dell 1996). These sources seem to be too far from the OB stars to be strongly ionized, although they are still likely bona fide members of the Trapezium Cluster. The silhouettes show a range in shapes, from circular to elliptical to cigar-shaped, consistent with a family of disks seen at various orientation angles from face-on to near edge-on. These disks range from 50-1000 AU in diameter, and each case, a young (1-3 Myr), low-mass (0.3-1.5 $\left.M_{\odot}\right)$ central star is seen either directly or, in Orion 114-426, indirectly via faint polar reflection nebulae above and below the plane of the near edge-on disk. 
Since the silhouette disks are resolved, their structure can be probed directly, at least at the edges. Figure 3 shows model disks placed in silhouette against a bright background, and their major axis profiles compared to that of one source, Orion 183-405. The first point to notice is that the edge of the disk is resolved, i.e., a model disk with an abrupt edge results in a profile that is sharper than observed. Conversely however, the observed profile is sharper than predicted for standard disk models, with surface densities $\Sigma \propto r^{-p}$ and $p=0.75-1.5$, depending on the mass of the disk relative to the central star. The observed profile is in fact most consistent with a truncation at some radius followed by an exponential decay beyond.

It is possible that this exponential edge is simply an effect of disk evolution, independent of environment: time-dependent models predict the development of transition zones between an inner quasi-equilibrium disk and an outer infalling envelope (Saigo \& Hanawa 1997), or between disk material that is predominantly spiralling inward, and that which is moving outward, carrying away excess angular momentum (Hartmann, personal communication). It is also possible that the disks are truncated by their local environment, i.e., a hot $\mathrm{H}$ II region full of stars. First, the ionizing radiation and winds from the OB stars could evaporate and ablate the disks at some radius as presumably occurs for the ionized proplyds. Second, pressure equilibrium will be established at some radius (and thus density) between the cold, high-density disk material and the hot, low-density H II region. Third, a rotating star-disk system moving through the $\mathrm{H}$ II region would induce compression and stripping on the leading and trailing edges of the disk respectively, thus leading to truncation and distortion. Finally, interactions between pairs of star-disk systems moving within the cluster could truncate the disks (Clarke \& Pringle 1991; Heller 1995; Larwood et al. 1996). Indeed, recent models predict just the sort of exponential truncation seen in the silhouette disks (Hall et al. 1996). To clarify which mechanisms are responsible for disk truncation, further high-resolution multi-wavelength surface density profiles obtained with the HST will have to be compared with models incorporating parameters including the distance from $\theta^{1} \mathrm{OriC}$, $\mathrm{H}$ II region density and temperature, local stellar density, etc.

\section{Star formation in the M 16 elephant trunks}

The now familiar HST image of M 16, the Eagle Nebula, shows molecular cloud cores being photo-evaporated by OB stars in the NGC 6611 cluster to form tall pillars or "elephant trunks" (Hester et al. 1996). Small-scale crenellations on the pillars were dubbed "evaporating gaseous globules", or EGGs, an acronym which also implies that these globules are the hatching sites of young stars. If low-mass stars are indeed forming in these EGGs, 


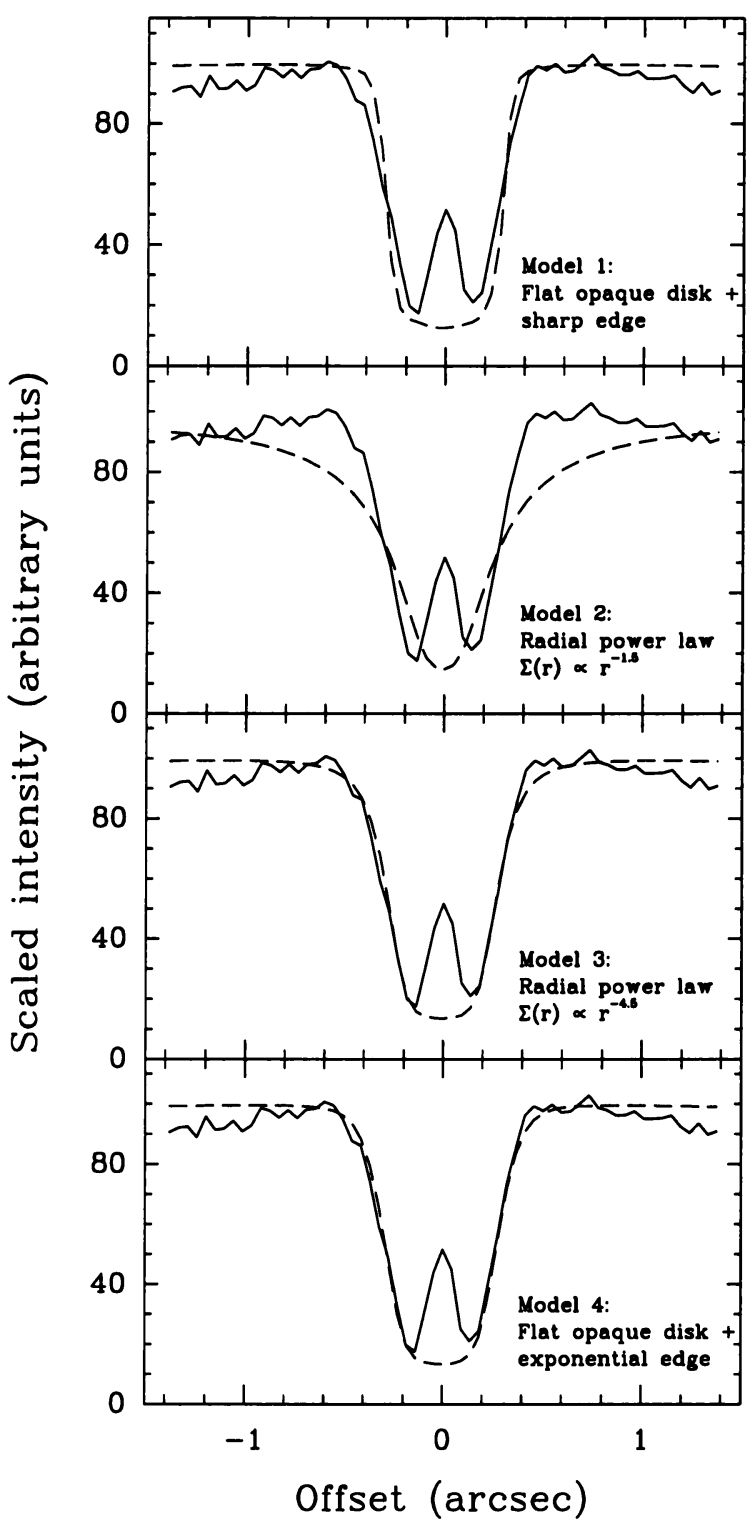

Figure 3. A major axis intensity profile across an [O III] image of the Orion 183-405 silhouette, including the central star (solid curve). Four disk models are superimposed (dashed curves): the model disk is thin, tilted at $45^{\circ}$, and convolved with a model HST+WFPC2 PSF to match the data. Model 1 is a completely opaque disk with sharp edges, and is too sharp to fit the observed disk edge. Model 2 has a canonical disk model power-law surface density profile with $\Sigma(r) \propto r^{-1.5}$ : now the model profile is much too shallow. Model 3 has a steeper power-law dependence, $\Sigma(r) \propto r^{-4.5}$, and while the fit is good, such a steep power-law is non-physical. Finally, Model 4 is an opaque central disk modified to have an exponentially decaying edge: this model fits well, and is physically the most plausible. 
there might be considerable ramifications, since the massive stars would apparently be responsible for terminating infall onto the low-mass stars, and thus determining the stellar masses and evolution of circumstellar disks. However, only limited evidence for stars embedded in the EGGs was presented by Hester et al. (1996): their HST continuum images showed one or two EGGs to have stars near their tips, while a few more coincidences were found in the near-IR images of Hillenbrand et al. (1994). However, the relatively poor sensitivity of the latter data limited the search to higher-mass stars $\left(>3 M_{\odot}\right)$, and the coarse image sampling $(1.3 \mathrm{arcsec} / \mathrm{pixel})$ made it difficult to be sure of the one-to-one correspondence between an IR source and an EGG: at near-IR wavelengths, the M 16 field is heavily contaminated by background field stars in the inner galactic bulge.

Clearly, more sensitive higher-resolution near-IR imaging is required. This has been attempted using a ground-based adaptive optics system (Currie et al. 1996), and images will also be taken with the HST under the NICMOS GTO program. However, both systems have a limited FOV (typically $\sim 20 \times 20$ arcsec), allowing only targeted observations of specific interesting EGGs to be carried out. This makes it difficult to carry out a statistically unbiased survey of all the EGGs, and equally importantly, prevents a comparably deep wide-field survey of the background population, a vital part of assessing field star contamination in this very crowded region.

We have carried out a multi-colour near-IR imaging survey of a $13 \times 13$ arcmin region of $\mathrm{M} 16$, covering the elephant trunks and a large surrounding area, including the nearby NGC 6611 cluster and the dense field star population in the galactic bulge beyond. Only the central section, corresponding to the WFPC2 FOV, is shown in Figure 4. The integration time of 2 minutes each at $J, H$, and $K^{\prime}$ gave $5 \sigma$ point source detection limits of $19^{\mathrm{m}} \cdot 2,18^{\mathrm{m}} \cdot 1$, and $17^{\mathrm{m}} \cdot 5$ respectively. The corresponding physical limits are complicated to define, since they are a product of the distance to M 16, the mass and age of a young star, and the extinction being penetrated. Roughly speaking however, $K^{\prime}=17^{\mathrm{m}} \cdot 5$ is equivalent to a $0.5 \mathrm{Myr}$ old, $0.5 M_{\odot}$ star at $2.0 \mathrm{kpc}$, seen through $A_{V} \sim 30^{\mathrm{m}}$. Assuming a typical diameter for an EGG of 1 arcsec or $2000 \mathrm{AU}$, this extinction corresponds to a constant uniform volume density of $\sim 2 \times 10^{6} \mathrm{~cm}^{-3}$, appropriate for a small dense cloud core. We have made a careful search for near-IR stars associated with the optical EGGs, aided by an accurate astrometric link between the two images (0.06 arcsec RMS). In the 73 EGGs of Hester et al. (1996), we have identified only 8 near-IR sources, i.e., roughly $10 \%$ of the EGGs have stars along their line-of-sight. Some of these are chance alignments with field stars: our measurements of the background field density indicate that two or three of the alignments will be coincidental. Therefore, roughly $7 \%$ of the EGGs are physically associated with low-mass stars down to $0.5 M_{\odot}$. 


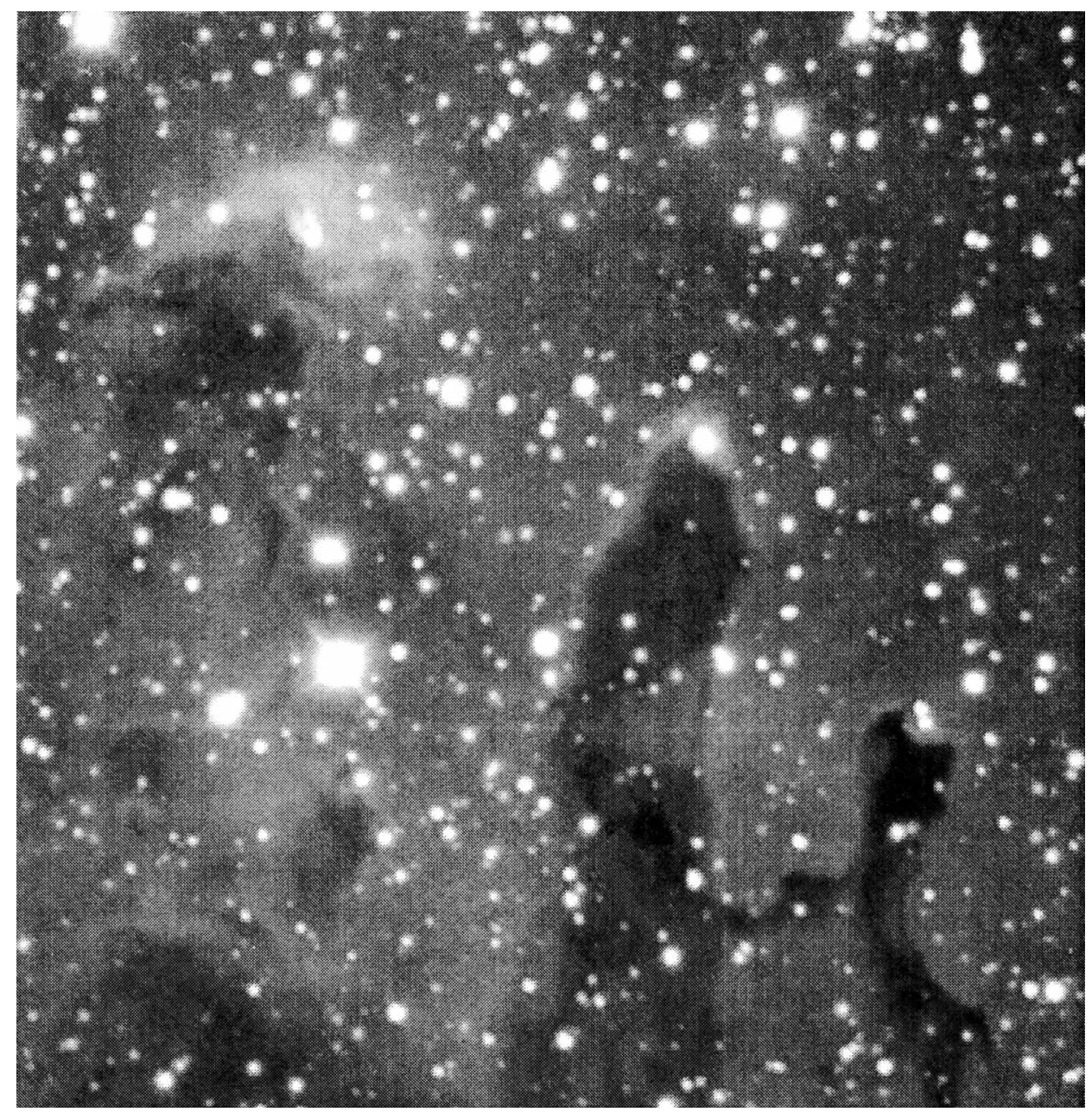

Figure 4. The elephant trunks in M16 at near-IR wavelengths. The image is a black-and-white rendering of a true-colour $J H K^{\prime}$ composite that only partly captures the almost transparent appearance of the elephant trunks and the dominant population of background field stars. The image covers roughly $1.5 \times 1.5 \mathrm{pc}$ at the $2.0 \mathrm{kpc}$ to $\mathrm{M} 16$. The data were obtained using Omega-Prime on the Calar Alto 3.5-m telescope in June 1996. With a $1024 \times 1024$ pixel $\mathrm{HgCdTe}$ array and an image scale of $0.4 \mathrm{arcsec} / \mathrm{pixel}$, the field is $6.7 \times 6.7$ arcmin: only a $2.5 \times 2.5$ arcmin extract is shown here.

Assuming a standard Miller-Scalo IMF, roughly one-third of all stars have masses $0.5 M_{\odot}$ or greater, and thus our survey could be missing two-thirds of an embedded low-mass population. Correcting for this, it is plausible that some $20 \%$ of all the EGGs might contain young stars.

Thus, the EGGs are indeed plausible sites of star formation, but only a minority of the EGGs appear to have been able to form stars before being 
disrupted. An interesting question is whether or not the stars have disks, following the suggestion by Hester etal. (1996) that the same ionization and winds from the OB stars in NGC 6611 responsible for illuminating and eroding the elephant trunks will also destroy any disks as soon as the young stars are exposed. In this regard, we are reminded of the ionized proplyds in Orion, and indeed, Hester et al. (1996) have suggested that the EGGs and proplyds are similar objects, simply viewed at different angles. This is a tricky issue to address here, but we can at least make two points. First, the EGGs and proplyds are different in one important regard: well over $90 \%$ of all proplyds in Orion do have associated near-IR stars, which cannot be said of the EGGs. Second, disks do appear to have survived in the ionized proplyds at least on small scales, as inferred from their near-IR excesses.

Nevertheless, the effects of environment are again found to be important in M 16: the OB stars ablate the columns, revealing the small population of embedded stars 'prematurely', and thus may play a role in determining the stars' masses (Hester et al. 1996). However, the limited WFPC2 FOV covered only a small and selective part of the region. The NGC 6611 cluster, centred $\sim 1 \mathrm{pc}$ to the north-west, contains thousands of low-mass stars that may have formed relatively quiescently before the OB stars turned on and disrupted their surroundings: in the elephant trunks, we may only be seeing 'secondary' star formation around the edges of the main cluster.

\section{Summary and prospects}

In the limited space available, we have only been able to briefly mention the effects of the external environment on star formation in the Orion and Eagle nebulae. The ionizing radiation and strong winds from the OB stars, as well as dynamical interactions in the dense cluster environment itself, appear to shape the evolution of low-mass stars and their circumstellar disks, truncating the reservoir for further accretion onto a star, thus perhaps setting its mass, and possibly destroying disks before planets have had a chance to form. Until recently, observations of such phenomena have been difficult, and it is only now possible to study the more distant star-forming regions in detail with the advent of high-spatial resolution optical, infrared, and millimetre instrumentation. The next generation of instrumentation including NICMOS on the HST, adaptive optics on 8-m class telescopes, SIRTF, FIRST, the NGST, and large sub-millimetre arrays promises further important insights. At the same time, theoretical work is beginning to take on the difficult task of understanding the complex energetic effects of high-mass stars on their low-mass neighbours, the effects of competition and interactions between the low-mass stars, and the cumulative effects on disks and planets around those stars. 
Acknowledgements: I would like to thank my collaborators, including John Bally, Bob O'Dell, John Stauffer, John Rayner, Hans Zinnecker, and Tom Hayward. I would also like to thank Jeff Hester for enlightening discussions and Bo Reipurth, Claude Bertout, et al. for organising the meeting.

\section{References}

Allen, L. E. 1996, PhD thesis, University of Massachussetts-Amherst

Bally, J., Devine, D., \& Sutherland, R. 1995, Rev. Mex. A. A. (Serie de Conferencias), 1,19

Clarke, C. J., \& Pringle, J. E. 1991, Mon. Not. R. astr. Soc., 249, 584

Currie, D., Kissell, K., Shaya, E., Avizonis, P., Dowling, D., \& Bonaccini, D. 1996, ESO Messenger, 86, 31

Felli, M., Taylor, G. B., Catarzi, M., Churchwell, E. B., \& Kurtz, S. 1993, Astr. Ap. Suppl., 101,127

Garay, G., Moran, J. M., \& Reid, M. J. 1987, Ap. J., 314, 535

Gomez, M., Hartmann, L. W., Kenyon, S. J., \& Hewett, R. 1993, A. J., 105, 1927

Hall, S. M., Clarke, C. J., \& Pringle, J. E. 1995, Mon. Not. R. astr. Soc., 278, 303

Hayward, T. L., \& McCaughrean, M. J. 1997, A. J., 113, 346

Heller, C. H. 1995, Ap. J., 455, 252

Henney, W. J., Raga, A. C., Lizano, S., \& Curiel, S. 1996, Ap. J., 465, 216

Herbig, G. H. \& Terndrup, D. M. 1986, Ap. J., 307, 609

Hester, J. J., et al. 1996, A. J., 111, 2349

Hillenbrand, L. A. 1997, A. J., in press

Johnstone, D., Hollenbach, D., Storzer, H., Bally, J., \& Sutherland, R. 1996, BAAS, 189, \#49.12

Kenyon, S. J., \& Hartmann, L. 1995, Ap. J. Suppl., 101, 117

Lada, E. A., Evans, N. J., II, De Poy, D. L., \& Gatley, I. 1991, Ap. J. (Letters), 371, 171

Lada, E. A., Strom, K. M., \& Myers, P. C. 1993, in Protostars and Planets III, eds. E. H. Levy \& J. I. Lunine, (Tucson: Univ. of Arizona Press), p245

Laques, P., \& Vidal, J.-L. 1979, Astr. Ap., 73, 97

Larwood, J., Nelson, R. P., Papaloizou, J. C. B., \& Terquem, C. 1996, Mon. Not. R. astr. Soc., 282, 597

Levy, E. H. \& Lunine, J. I. 1993, editors of Protostars \& Planets III, (Tucson: Univ. of Arizona Press)

McCaughrean, M. J., \& Stauffer, J. R. 1994, A. J., 108, 1382

McCaughrean, M. J., Rayner, J. T., Zinnecker, H., \& Stauffer, J. R. 1996, in Disks and Outflows around Young Stars, eds. S. V. W. Beckwith, J. Staude, A. Quetz, \& A. Natta, (Heidelberg: Springer), p33

McCaughrean, M. J., \& O'Dell, C. R. 1996, A. J., 111, 1977

Mundy, L. G., Lada, E. A., \& Looney, L. 1995, Ap. J., 452, 137

O'Dell, C. R., Wen, Z., \& Hu, X. 1993, Ap. J., 410, 696

O'Dell, C. R., \& Wen, Z. 1994, Ap. J., 436, 194

O'Dell, C. R., \& Wong, S.-K. 1996, A. J., 111, 846

Prosser, C. F., Stauffer, J. R., Hartmann, L., Soderblom, D. R., Jones, B. F., Werner, M. W., \& McCaughrean, M. J. 1994, Ap. J., 421, 517

Saigo, K., \& Hanawa, T. 1997, in Low Mass Star Formation from Infall to Outflow, eds. F. Malbet \& A. Castets, IAU Symposium 182, p247

Stauffer, J. R., Prosser, C. F., Hartmann, L., \& McCaughrean, M. J. 1993, A. J., 108, 1375

Strom, K. M., Strom, S. E., \& Merrill, K. M. 1993, Ap. J., 412, 233

Zinnecker, H., McCaughrean, M. J., \& Wilking, B. A. 1993, in Protostars and Planets III, eds. E. H. Levy \& J. I. Lunine, (Tucson: Univ. of Arizona Press), p429 